\title{
Electronic Properties of Polymer Crystals: The Effect of Interchain Interactions
}

\author{
Andrea Ferretti, Alice Ruini, and Elisa Molinari \\ INFM National Center on nanoStructures and bioSystems at Surfaces $\left(S^{3}\right)$ and Dipartimento di Fisica, \\ Università di Modena e Reggio Emilia, Via Campi 213/A, 41100 Modena, Italy \\ Marilia J. Caldas \\ Instituto de Física, Universidade de São Paulo, Cidade Universitária, 05508-900 São Paulo, Brazil and INFM-S ${ }^{3}$, Italy
}

(Received 24 July 2002; published 25 February 2003)

\begin{abstract}
We present a theoretical study of the transport parameters in a prototype conjugated-polymer, polypara-phenylenevinylene, in two different possible crystalline packings. Our analysis is performed through density-functional electronic structure calculations, and allows one to obtain the fundamental parameters describing charge transport. The transfer integrals are found to be a crucial quantity to appreciate the effects of crystalline aggregation on conduction properties: our results indicate that interchain interactions can be viewed as a tunable parameter for the design of efficient electronic devices based on organic materials.
\end{abstract}

PACS numbers: 71.20.Rv, 71.70.-d

The investigation of charge transport in complex organic materials is a huge challenge for both theory and experiment. This is the case, for example, for the active conjugated-polymer films in electronic and optoelectronic devices [1], and for electron transfer between donor and acceptor sites in proteins $[2,3]$.

Control of carrier mobility is indeed a basic need for organic devices design, and one would expect clear experimental data and interpretation to be available for the most studied materials, such as poly-para-phenylenevinylene (PPV). In the case of long conjugated polymers, with delocalized $\pi$-electron structure, the main transport path is known to be intramolecular: this is reflected in the high electrical anisotropy seen for stretched material [4]. This is not the case for films made from oligomers, where transport orthogonal to the long molecular axis could dominate; it has been shown that for PPV the film morphology strongly influences the transport properties, indicating that intramolecular interactions are not sufficient to explain experiments [5]. As most polymer films grown by coating or casting from solution are disordered [6], realistic models of transport properties in such configurations would be essential. In polymeric systems this requires inclusion of two essential components usually described as "intrachain" and "interchain" charge transport. A similar situation exists in the case of proteins [3], where the existence of intrinsically different paths for electron transfer has been described as "through bonds" or "through space."

The main quantity of interest is the transfer integral (TI) $E_{i j}$ between "sites" $i, j$, to be inserted, e.g., in a Büttiker-Landauer [7-9] expression for conductivity [10,11] or in the Marcus [2] formulation for electron transfer. In order to predict the impact of a specific chain packing we must obtain reliable values for the relevant TI.

This task can best be performed for simple model systems that can be tested against experiment while retaining the main properties of the samples of interest, namely, the three-dimensional (3D) character of onedimensional (1D) weakly interacting systems. Very recently, extremely ordered crystalline films of some oligomeric materials have been achieved [12], opening the way to the comparison with accurate theoretical studies. These can now be performed through $a b$ initio density-functional theory (DFT) methods, using the solid-state physics implementations that provide reliable microscopic information on structural and electronic properties of extended systems [13].

In this Letter we investigate and compare interchain TI for two typical morphologies shown by PPV: herringbone and displaced $\pi$ stacks (HB and $\pi \mathrm{S}$; see Fig. 1). The first is attained in crystalline pure PPV [16], while the stack is assumed by several derivatives of the polymer, for example, by poly[2-methoxy, 5-(2'-ethyl-hexyloxy) phenylene vinylene] (MEH-PPV) [17]. Our results are based on first-principles calculations for the band structure of model crystalline systems, from which we directly extract effective masses and TI for transport orthogonal to the chain direction. We find that although the transverse effective masses are similar for both crystal structures, the TI are much more sensitive to the different interchain environments. The TI is 1 order of magnitude larger for $\pi$ stacks in the stack direction than for herringbone packing; this indicates that 3D arrangement can be indeed a crucial element for the design of materials with efficient transport properties.

We first obtain the full band structure of the model crystal through first-principles DFT calculations and then convert the problem to a tight-binding formulation, in a Slater-Koster spirit [18], by exact discrete Fourier transforms. Our ansatz is that the evolution of the highest occupied states in a $\mathbf{k}$ plane (i.e., a plane in reciprocal space) orthogonal to the chain direction $\hat{z}$ can be treated as coming from one (highest occupied) molecular orbital 

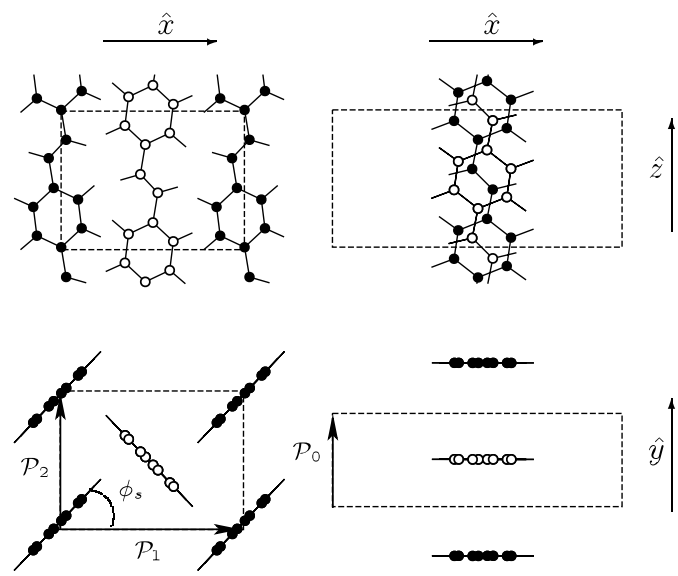

(a) $\mathrm{HB}$

(b) $\pi \mathrm{S}$

FIG. 1. Representation of chain arrangement in the crystal: (a) PPV herringbone: monoclinic unit cell, $a=8.07 \AA(\hat{x}), b=$ $6.05 \AA, c=6.54 \AA(\hat{z}), \hat{\mathbf{b c}}=123^{\circ}$, setting angle $\phi_{s}=52^{\circ}$ [14]. (b) Displaced $\pi$ stack: base-centered orthorombic bravais lattice, $a=15.00 \AA(\hat{x}), b=7.12 \AA(\hat{y}), c=6.65 \AA(\hat{z})$, with one chain per unit cell, interchain distance in the stack direction $3.56 \AA$ [15]. We show in the upper part the relative shift of the chains by half a unit cell in $\hat{z}$ direction. The $\hat{x}$ separation is enough to isolate the chains, simulating aliphatic side chains.

(HOMO) per chain. This is justified by the much smaller dispersion of the HOMO band in that $\mathbf{k}$ plane, compared to the $\hat{z}$ dispersion, and by the similarity of the crystalline HOMO to the single-chain HOMO. A corresponding ansatz applies to the lowest unoccupied molecular orbitals (LUMO) and states.

In Fig. 2 we report ab initio energy band structures $\epsilon(\mathbf{k})$ for both $\mathrm{HB}$ and $\pi \mathrm{S}$ systems as a function of the wave vector $\mathbf{k}$ along relevant directions of the Brillouin zone (BZ) (see Fig. 3, lower panels). The calculations are performed within the local density approximation (LDA) for the exchange-correlation potential, using a plane-

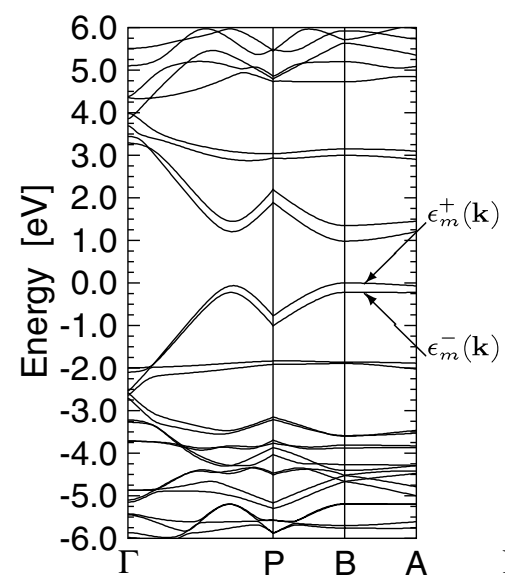

(a) $\mathrm{HB}$

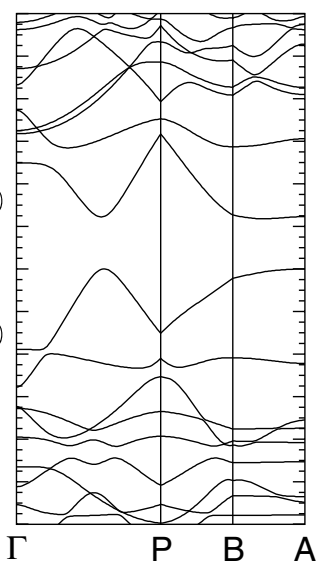

(b) $\pi \mathrm{S}$
FIG. 2. LDA band structures for the HB and $\pi \mathrm{S}$ structures. Energies in eV relative to the valence band top. Symmetry labels as in Fig. 3, following Ref. [19]. wave basis set and nonlocal pseudopotentials through the PWSCF code [20]. We do not correct band structures to quasiparticle energies, since band dispersions and effective masses were found to be well described within LDA for similar systems [21,22]. We note that the HB structure shows a splitting of the bands coming from the presence of two symmetry-inequivalent chains in the unit cell (also known as Davydov splitting [23]), while the $\pi \mathrm{S}$ structure does not. Apart from that there is a noticeable similarity of the band structure in the directions shown. In particular, the HOMO and LUMO bands are well separated from the other bands (except for the anticrossing with a flat band far from the band edges). The largest dispersion occurs for the chain direction $\hat{z}$, but one can observe that the dispersion orthogonal to $\hat{z}$ is nonnegligible, indicating interchain interaction.

We now look in more detail at the band structure orthogonal to $\hat{z}$, for the HOMO states. Since the bands are very flat in any $\mathbf{k}$ plane orthogonal to $\hat{z}$, it is instructive to view a $\mathbf{k}$ plane including the $z$ axis and to analyze the behavior of the HOMO band as we move away from it. We show in Fig. 3 the energies in chromatic scale for the $y z$ plane, which includes the top of the valence band in the $\mathrm{HB}$ case (for $\pi \mathrm{S}$ the bands are flat along $\hat{x}$, reflecting the thorough isolation of the stacks, so the $\mathrm{BZ}$ is to all effects two dimensional as shown in the figure). In the
HB

(a)

(c)
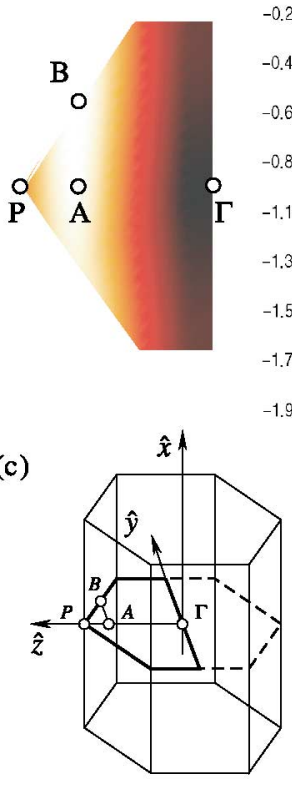

-1.77
-1.99

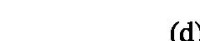

(d)

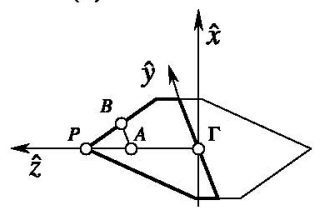

FIG. 3 (color). Surface plots of energy bands and BZ definitions: (a) and (b) refer to HOMO bands for $\mathrm{HB}$ and $\pi \mathrm{S}$, respectively. BZ for these systems are shown in (c) and (d), highlighting the $\mathbf{k}$ planes used in (a) and (b). High-symmetry and relevant $\mathbf{k}$ points used in Fig. 2 are marked: chain direction is along the $\Gamma-P$ line. The top of the valence band coincides with $B$ for the HB structure (a), and with $A$ for the $\pi \mathrm{S}$ case (b). Energies in $\mathrm{eV}, \mathbf{k}$ units are coherent within plots (a) and (b). 
noninteracting limit, we should obtain completely planar energy fronts, orthogonal to $\hat{z}$. However, we see a distinct curvature of the high-energy ridge for $\pi \mathrm{S}$, not seen for HB.

The effective masses are calculated at the top of the valence (HOMO) and bottom of the conduction (LUMO) bands, accounting for the full 3D tensor. The principal axes of the inverse mass tensor numerically coincide with our $\hat{x}, \hat{y}, \hat{z}$ axes for both systems: the resulting effective mass parameters are reported in Table I. These data show the general tendency of LUMO bands to be more dispersive in the $\hat{y}$ direction; electrons have lower effective masses than holes. Masses along the chain backbones are quite similar for both systems, which indicates at this level that the details of crystalline packing do not play a relevant role in this direction. Nevertheless, effective masses in directions orthogonal to the chains are only between 1 and 2 orders of magnitude larger, thus indicating an important contribution of solid-state effects.

An analysis based only on the effective masses is not able to account for differences between packing structures (HB and $\pi \mathrm{S}$ ), such as those shown in Fig. 3: effective masses are local parameters in $\mathbf{k}$, pertaining to the absolute maximum (minimum) of the band, and relevant to the low-doping, low-temperature limit. In the case of polymer or oligomer films, we are usually in an intermediate-to-high doping regime, and we should more properly work with the whole band structure.

We now move on to the study of TI that reflect the whole band curvature, and can give us information on specific neighborhood interactions. There are two fundamentally different periodicities for polymer crystals: the periodicity of each single chain along the chain axis, described by the lattice vector c, and the (interchain) two-dimensional periodicity, which we denote by $\mathcal{P}$. We can explicitly acknowledge the weak interaction on $\mathcal{P}$ and write the generic eigenstate $\psi_{l \mathbf{k}}$ of the crystal Hamiltonian as

$$
\psi_{l \mathbf{k}}(\mathbf{r})=\sum_{s, m, j} C_{m, j}^{l} e^{i \mathbf{k} \cdot \mathcal{P}_{s}} \phi_{m k_{z}}^{j}\left(\mathbf{r}-\mathcal{P}_{s}-\boldsymbol{\tau}_{j}\right),
$$

where the sum is over cells $(s)$, inequivalent chains in the unit cell $(j)$, and, if necessary, isolated chain band index $(m)$. The $\phi$ 's are the solutions of the single-chain eigenvalue problem: the noteworthy feature in Eq. (1) is that the only $\mathbf{k}$ state of the isolated chain structure contributing to the expansion is that corresponding to the com-

TABLE I. Effective masses in units of the free-electron mass.

\begin{tabular}{lcccc}
\hline \hline & & HB & \multicolumn{2}{c}{$\pi \mathrm{S}$} \\
& HOMO & LUMO & HOMO & LUMO \\
\hline$\hat{x}$ & 3.54 & 2.52 & $\infty$ & $\infty$ \\
$\hat{y}$ & 5.24 & 1.31 & 5.56 & 2.74 \\
$\hat{z}$ & 0.11 & 0.11 & 0.10 & 0.10 \\
\hline \hline
\end{tabular}

086401-3 ponent of $\mathbf{k}$ along the chain direction $k_{z}=\mathbf{k} \cdot \hat{z}$. One can thus arrive at the following definition of TI:

$$
E_{m i, n j}^{k_{z}}\left(\mathcal{P}_{s}\right)=\int d^{3} \mathbf{r} \phi_{m k_{z}}^{*}\left(\mathbf{r}-\boldsymbol{\tau}_{i}\right) H \phi_{n k_{z}}\left(\mathbf{r}-\mathcal{P}_{s}-\boldsymbol{\tau}_{j}\right)
$$

As seen from the band structures in Fig. 2, we can consider only one state per chain in the unit cell, the HOMO or the LUMO. Here we will focus on the hole transport and the HOMO states. For the simple case of one chain per unit cell $(\pi \mathrm{S})$, assuming zero overlap between the states of different chains, the interchain TI can be obtained for an arbitrary number of neighbors through exact discrete Fourier transforms of the band along the orthogonal direction:

$$
E_{m}^{k_{z}}\left(\mathcal{P}_{s}\right)=\frac{1}{Q} \sum_{q=1}^{Q} e^{-i \mathbf{k}_{q} \cdot \mathcal{P}_{s}} \epsilon_{m}\left(\mathbf{k}_{q}\right)
$$

where $Q$ is defined by the number of neighbors at $\mathcal{P}_{s}$ taken into account.

When by crystal symmetry there are two inequivalent $(j=1,2)$ chains per unit cell, the TI must be obtained from a $2 \times 2$ matrix; taking into account the improper rotation bringing one chain into the other in the HB case, the following relations hold:

$$
\begin{gathered}
\sum_{s} e^{i \mathbf{k} \cdot \mathcal{P}_{s}} E_{m 1, m 1}^{k_{z}}\left(\mathcal{P}_{s}\right)=\frac{1}{2}\left[\epsilon_{m}^{+}(\mathbf{k})+\epsilon_{m}^{-}(\mathbf{k})\right], \\
\left|\sum_{s} e^{i \mathbf{k} \cdot \mathcal{P}_{s}} E_{m 1, m 2}^{k_{z}}\left(\mathcal{P}_{s}\right)\right|=\frac{1}{2}\left[\epsilon_{m}^{+}(\mathbf{k})-\epsilon_{m}^{-}(\mathbf{k})\right],
\end{gathered}
$$

where $\boldsymbol{\epsilon}_{m}^{+}(\mathbf{k})\left[\boldsymbol{\epsilon}_{m}^{-}(\mathbf{k})\right]$ is the maximum (minimum) diagonal element of the $2 \times 2$ matrix. From Eq. (4) it is possible to obtain the TI for equivalent chains through a simple Fourier transform. In order to calculate TI between inequivalent chains [Eq. (5)] we make the further simplifying assumption of retaining nearest neighbors only (the next interaction would be between third neighbors).

Our results are reported in Table II, and we now see a large difference emerging between the structures, with the nearest-neighbor coupling in the $\pi \mathrm{S}$ structure more that 4 times larger than any coupling in the HB crystal. Surprisingly, the stronger coupling in the HB structure arises for the (nearest-neighbor) nonequivalent chains, in

TABLE II. Absolute values of transfer integrals of HOMO states in meV. For clarity we use $E_{i j}\left(\mathcal{P}_{s}\right)$ in place of $E_{m i, m j}^{k_{z}}\left(\mathcal{P}_{s}\right)$, since $m$ refers always to the top valence band, and $k_{z}$ is set to the $z$ component of the HOMO $\mathbf{k}$ vector.

\begin{tabular}{crrr}
\hline \hline HB & & & $\pi \mathrm{S}$ \\
\hline$E_{12}(0)$ & 27.42 & $E\left(\mathcal{P}_{0}\right)$ & 120.64 \\
$E_{11}\left(\mathcal{P}_{2}\right)$ & 13.96 & $E\left(2 \mathcal{P}_{0}\right)$ & 10.49 \\
$E_{11}\left(\mathcal{P}_{1}+\mathcal{P}_{2}\right)$ & 1.49 & $E\left(3 \mathcal{P}_{0}\right)$ & 2.86 \\
$E_{11}\left(\mathcal{P}_{1}\right)$ & 0.27 & & \\
\hline \hline
\end{tabular}


spite of the large interchain angle. The "stacklike" coupling along $\mathcal{P}_{1}$ is prevented by the increased distance, and along $\mathcal{P}_{2}$ by the large lateral displacement of one chain relative to the neighbors. This latter finding is in good agreement with ab initio results for other organic systems [24] that highlight the relevance of the precise stacking geometry. It is interesting to compare results for the TI along the chain direction, for the case of an isolated chain. Now we consider the HOMO states of the monomer as generating orbitals for the band, in a similar spirit as above; we are again justified by the clear origin of the HOMO band in these systems. We find that the TI for nearest neighbors is in that case $E(\mathbf{c})=523 \mathrm{meV}$, and thus transport in the stack direction for $\pi \mathrm{S}$ films will have efficiencies of the same order of magnitude as for long stretched polymer chains along the chain direction.

Recently, semiempirical calculations for selected aggregates of similar oligomers were performed [25], assuming ad hoc that the nearest-neighbors-only approximation could be adopted. Our results show that for the HB structure this hypothesis is quite reasonable; however, this is not the general case, as shown for the $\pi \mathrm{S}$ structure. This could be investigated only because we modeled a fully periodic system.

We learn from this analysis that, for the HB structure, coupling orthogonal to the chain direction is much weaker than along the chain, supporting the wealth of models that completely neglect interchain-hopping contributions to transport in long-polymer films. However, it has been accepted that the long aliphatic side chains of, e.g., MEH-PPV were responsible mainly for preventing interaction between chains, thereby preserving the properties of the isolated chain. What we see is that the organization in $\pi$ stacks instead promotes interchain interactions, and on the whole should increase the hole conductivity of the material. This feature, together with the absence of Davydov splitting (responsible for luminescence quenching [26] in HB-PPV), sums up to better device performance expected for highly organized $\pi \mathrm{S}$ films. We did not take into account polaron relaxation which should occur in real samples. However, for PPV, the relaxation is associated with slight quinoid distortion of the rings, and charge localization [25]. Since the distortion does not affect interchain angles and general pointsymmetry properties, the values we obtain for TI should be appropriate to the closely packed regions of real samples.

In conclusion, we have shown that interchain interactions are very sensitive to the specific 3D structure, and can thus be used to tailor the transport properties of conjugated-polymer films.

We are grateful to G. Bussi and A. Calzolari for stimulating discussions. Calculations partly done at CINECA under an INFM grant. We gratefully acknowledge support by the EU Network "Exciting" and by the Italian Ministry for Foreign Affairs (MAE). M. J.C. acknowledges support from FAPESP and CNPq (Brazil).

086401-4
[1] J. H. Burroughes, D. D. C. Bradley, A. R. Brown, R. N. Marks, K. Mackay, R. H. Friend, P. L. Burns, and A. B. Holmes, Nature (London) 347, 539 (1990).

[2] R. A. Marcus and N. Sutin, Biochim. Biophys. Acta 811, 265 (1985).

[3] V. S. Pande and J. N. Onuchic, Phys. Rev. Lett. 78, 146 (1997).

[4] M. Ahlskog, M. Reghu, A. J. Heeger, T. Noguchi, and T. Ohnishi, Phys. Rev. B 53, 15529 (1996).

[5] T. Q. Nguyen, R. C. Kwong, M. E. Thompson, and B. J. Schwartz, Appl. Phys. Lett. 76, 2454-2456 (2000).

[6] N. Greenham and R. Friend, in Solid State Physics, edited by $\mathrm{H}$. Ehrenreich and F. Spaepen (Academic, San Diego, CA, 1995), pp. 2-150.

[7] R. Landauer, Philos. Mag. 21, 863 (1970).

[8] M. Büttiker, Y. Imry, R. Landauer, and S. Pinhas, Phys. Rev. B 31, 6207 (1985).

[9] M. Büttiker, Phys. Rev. Lett. 57, 1761 (1986).

[10] P. A. Schulz, D. S. Galvão, and M. J. Caldas, Phys. Rev. B 44, 6073 (1991).

[11] R. Hey, F. Gagel, M. Schreiber, and K. Maschke, Phys. Rev. B 55, 4231 (1997).

[12] See, e.g., F.-J. M. zu Heringdorf, M. C. Reuter, and R. M. Tromp, Nature (London) 412, 517 (2001); C. Kloc, P. G. Simpkins, T. Siegrist, and R. A. Laudise, J. Cryst. Growth 182, 416 (1997), and references therein.

[13] See, e.g., R. O. Jones and R. Gunnarsson, Rev. Mod. Phys. 61, 689 (1989); M. C. Payne, M. P. Teter, D. C. Allan, T. A. Arias, and J. D. Joannopoulos, Rev. Mod. Phys. 64, 1045 (1992), and references therein.

[14] D. Chen, M. J. Winokur, M. A. Masse, and F. E. Karasz, Phys. Rev. B 41, 6759 (1990).

[15] C. Y. Yang, F. Hide, M. A. Díaz-García, A. J. Heeger, and Y. Cao, Polymer 39, 2299 (1998).

[16] D. Chen, M. J. Winokur, M. A. Masse, and F. E. Karaz, Polymer 33, 3116 (1992).

[17] E. M. Conwell, J. Perlstein, and S. Shaik, Phys. Rev. B 54, R2308 (1996).

[18] J. C. Slater and G. F. Koster, Phys. Rev. 94, 1498 (1954).

[19] P. Gomes da Costa, R. G. Dandrea, and E. M. Conwell, Phys. Rev. B 47, 1800 (1993).

[20] S. Baroni, A. Dal Corso, S. de Gironcoli, and P. Giannozzi, http://www.pwscf.org (2001).

[21] J.-W. van der Horst, P. A. Bobbert, M. A. J. Michels, G. Brocks, and P. J. Kelly, Phys. Rev. Lett. 83, 4413 (1999).

[22] M. Rohlfing, M. L. Tiago, and S. G. Louie, Synth. Met. 116, 101 (2001)

[23] A. S. Davydov, Theory of Molecular Excitons (McGrawHill, New York, 1962).

[24] A. Calzolari, R. Di Felice, E. Molinari, and A. Garbesi, Appl. Phys. Lett. 80, 3331 (2002).

[25] J. L. Brédas, D. Beljonne, J. Cornil, J. P. Calbert, Z. Shunai, and R. Silbey, Synth. Met. 125, 107 (2002).

[26] A. Ruini, M. J. Caldas, G. Bussi, and E. Molinari, Phys. Rev. Lett. 88, 206403 (2002). 